
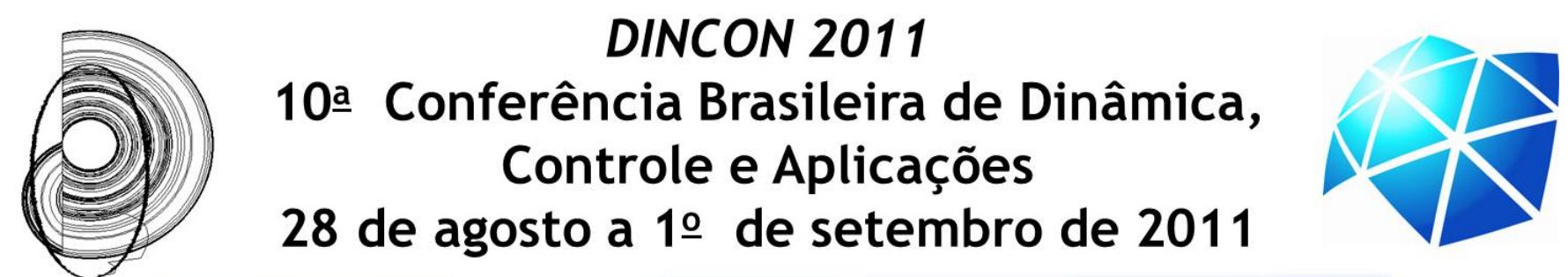

\title{
Attitude Improvement and Angular Rate Estimation using a Kalman Filter with Measurements of Four Tetrahedrally Arranged Low Cost MEMS Gyros
}

\author{
Francisco Granziera Júnior ${ }^{1}$, Hélio K. Kuga ${ }^{2}$, Marcelo C. Tosin ${ }^{3}$ \\ ${ }^{1}$ INPE, São José dos Campos/SP, Brasil, francisco.granzierajr@gmail.com \\ ${ }_{2}^{2}$ INPE, São José dos Campos, SP, Brazil, hkk@dem.inpe.br \\ ${ }^{3}$ State University of Londrina, Londrina/PR, Brasil, mctosin@uel.br
}

\begin{abstract}
This work presents the simulation of an angular velocity estimation system composed by four tetrahedrally arranged MEMS gyrometers. The timewise angular velocity of the sensor's readings are transformed to tri-orthogonal measurement sets by a pseudo-inverse matrix. A Kalman Filter utilizes periodically received attitude data to estimate the sensor's bias and also a new attitude. Also, the angular velocity readings are used to propagate the system's state until arrival of the next attitude information. The Kalman Filter estimation and propagation equations used in this process are presented in the paper. Also, a Monte Carlo simulation results are shown demonstrating the filter's convergence. This procedure will be implemented in an attitude determination device that will be integrated as an experiment aboard ITASAT-1 university satellite.
\end{abstract}

Keywords: MEMS Sensors, ITASAT, Kalman Filter.

\section{INTRODUCTION}

MEMS (Micro-Electro-Mechanical-Systems) are finding their way into inertial applications where typically big, heavy and expensive sensors had been used. Automobiles, airplanes, ships, missiles and artificial satellites are good examples of applications, but the last one is the most critical because mass, volume and power consumption are very restrictive topics for space applications. Nowadays, mechanical or optic fiber gyros are irreplaceable items of high accuracy inertial systems, because MEMS technology hasn't reached the necessary performance. This technology has been driven by applications in low cost consumer electronics in the last decade and inertial sensors, such as accelerometer and gyrometers, are commonly found, for example, in cell phones, cars, gaming human interfaces and others. Due to a growing demand on consumer products, especially on car applications, MEMS inertial sensors had evolved into another performance class. It is now able to drive, also, entry level inertial grade systems with applications on military, aeronautical and space markets, such as pico, nano and micro satellites. In the application of MEMS sensors the processing done on its data is almost mandatory [1]. One simple procedure that can greatly enhance the quality of the measurement is to perform a previous calibration on the sensor and then a re-calibration on the run [2]. The objective of this pre-calibration is to find the constant parameters of the measurement model. The basic components of a sensor model, considering it linear, are the scale factor and bias. When dealing with an array of sensors the model also depends on the alignment errors of the sensors. Some models consider that these parameters are constant or even temperature dependant and this is not accurate for MEMS sensors. A MEMS gyro has a bias which model could be approximated to a constant value added to a signal variation or noise which resembles a random walk, ie, it will never be completely determined [3]. To mitigate this problem, the utilization of gyros to read angular velocities reinforces the use of a bias estimator, which is only possible to be implemented with external attitude inferences (information of attitude given by reference sensors). With this complementary information it is possible to estimate the system's angular velocity and compare it with that one provided by the gyros. The difference between these measurements is considered the bias. This work describes a Kalman Filter that utilizes the readings of a set of four tetrahedrally arranged MEMS gyrometers together with external attitude information (provided by reference sensors, for example) to continuously estimate the angular velocity information bias and improve the attitude determination and its propagation. This system is simulated using a Monte Carlo technique and its results are discussed showing the convergence of the estimated bias and attitude. The purpose of this paper is to describe a preliminary study of an algorithm to determine the attitude of a satellite. This is part of an experiment carried by the ITASAT-1 university micro-satellite [4]. The experiment is composed by a set of MEMS gyrometers and has digital processing capability. It combines the external attitude data from the satellite with the gyrometers' data to calculate and provide improved attitude data. The external attitude information and its statistics will be provided by the satellite's attitude control system (ACS), which will be calculated, for instance, from magnetometers and sun sensors.

\section{MEASUREMENTS MODELS}

\subsection{The Gyros}

The sensors are grouped in a way that their measurement axes are perpendicular to the faces of a regular tetrahedron. This configuration, besides reducing the sensor readings errors, also allows the detection and tolerance of a failure in 
one of the sensors. Fig. 1 illustrates the measurement axes $\left(\mathrm{S}_{\mathrm{i}}\right)$ of this sensor arrangement.

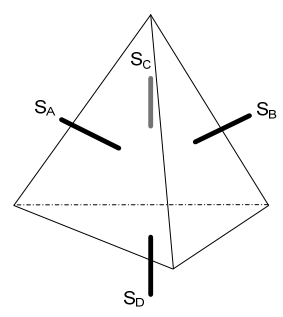

Fig. 1: Tetrahedrally displaced four sensor arrangement.

The relation between the sensor measurement axes and the body frame, which is orthogonal, is given by:

$$
\mathbf{s}=\mathbf{W u} \quad \therefore\left[\begin{array}{l}
s_{1} \\
s_{2} \\
s_{3} \\
s_{4}
\end{array}\right]=\left[\begin{array}{ccc}
2 \sqrt{2} / 3 & 0 & 1 / 3 \\
-\sqrt{2} / 3 & \sqrt{6} / 3 & 1 / 3 \\
-\sqrt{2} / 3 & -\sqrt{6} / 3 & 1 / 3 \\
0 & 0 & 1
\end{array}\right]\left[\begin{array}{l}
u_{x} \\
u_{y} \\
u_{z}
\end{array}\right]
$$

The pseudo-inverse of matrix $\mathrm{W}$ allows an estimate of the read velocities $\mathbf{u}=\left(\mathbf{W}^{\mathrm{T}} \mathbf{W}\right) \mathbf{W}^{\mathrm{T}} \mathbf{s}$. Due to the fact that sensors data in $\mathbf{s}$ have bias and noise, the vector $\mathbf{u}$ also presents this type of error. The true angular velocity can be defined as the measurement $\mathbf{u}$ subtracted from the bias $\mathbf{b}$ and noise $\boldsymbol{\eta}$, described by $\boldsymbol{\omega}=\mathbf{u}-\mathbf{b}-\boldsymbol{\eta}$.

\subsection{The Attitude Information}

The ACS provides attitude information formatted as unitary quaternion at a rate not superior to $1 \mathrm{~Hz}$. Besides the attitude information, for the Kalman Filter correct operation, it also requires the attitude covariance data.

\section{DATA HANDLING}

\subsection{Dynamic's Equations}

The Kalman Filter design requires a model for the states dynamics. The state is defined as the attitude quaternion and the bias vector transformed to the orthogonal base:

$$
\mathbf{x}=\left[\begin{array}{l}
q \\
\mathbf{b}
\end{array}\right]=\left[\begin{array}{lllllll}
q_{1} & q_{2} & q_{3} & q_{0} & b_{1} & b_{2} & b_{3}
\end{array}\right]^{\mathrm{T}}
$$

The dynamic equations for the quaternion and bias are given by [5-6]:

$$
\begin{aligned}
& \&(t)=1 / 2 \cdot \omega(t) \otimes q(t)=1 / 2 \cdot \mathbf{\Omega}_{\mathbf{4}}\left(\mathbf{u}-\mathbf{b}-\boldsymbol{\eta}_{1}\right) \stackrel{q}{q} \\
& \dot{\theta}(t)=1 / \tau \cdot \mathbf{b}(t)+\boldsymbol{\eta}_{2} \approx \boldsymbol{\eta}_{2}
\end{aligned}
$$

In the first equation, the quaternion derivative is written initially as the quaternionic product between the pure quaternion (without the scalar part) describing the angular velocity and the attitude quaternion. In terms of matrices it is possible to write the same expression using the skewsymmetric operator $\Omega_{4}$, which has dimension four, multiplied by the quaternion vector. In the second equation, the first term can be suppressed if $\tau$ is too big. The noises $\boldsymbol{\eta}$ are considered uncorrelated and Gaussian with zero mean.

Now, based on these dynamic equations and on the measurement models, it is possible to define the Kalman Filter's propagation and estimation stages.

\subsection{Description of the Kalman Filter}

Fig. 2 shows the mechanism to implement the Kalman Filter. The filter is partitioned in two steps: propagation and estimation. Note that input data are the data from gyros converted to the orthogonal form, the attitude from the satellite and their respective covariances.

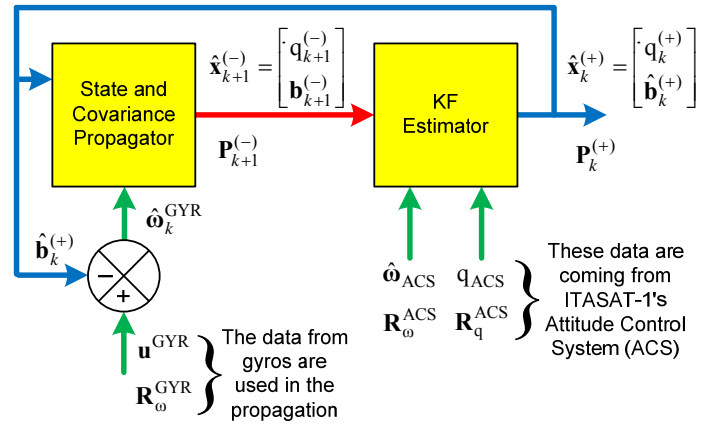

Fig. 2: Kalman Filter used to fuse ACS attitude and gyros data.

\subsubsection{State and Covariance Propagation}

In the propagation step, the previous estimated bias vector is subtracted from the gyros' data, resulting in an estimate of angular velocity:

$$
\hat{\boldsymbol{\omega}}_{k}^{\mathrm{GYR}}=\mathbf{u}_{k}^{\mathrm{GYR}}-\hat{\mathbf{b}}_{k}^{(+)}
$$

Integrating this velocity results in a small angular increment. If the time interval between samples is small enough, this increment can be computed as the product of angular velocity and this time interval:

$$
\Delta \boldsymbol{\theta}=\hat{\boldsymbol{\omega}}_{k}^{\mathrm{GYR}} \Delta t
$$

This angular increment can be expressed as a small incremental rotation which updates the quaternion through a quaternion product. Otherwise the incremental quaternion can be written in matrix form as:

$$
\mathbf{M}(\Delta \boldsymbol{\theta})=\cos (|\Delta \boldsymbol{\theta}| / 2) /|\Delta \boldsymbol{\theta}| \cdot \mathbf{I}_{4}+\sin (|\Delta \boldsymbol{\theta}| / 2)|\Delta \boldsymbol{\theta}| \cdot \mathbf{\Omega}_{4}(\Delta \boldsymbol{\theta})
$$

Matrix $\mathbf{I}_{4}$ is an identity matrix and matrix $\Omega_{4}$ is a dimension 4 skew-symmetric matrix with zeros in its diagonal. From the quaternion propagation matrix, defined in Eq. 6, it is possible to propagate the filter state using the relationship:

$$
\left[\begin{array}{l}
\mathfrak{q}_{k+1}^{(-)} \\
\hat{\mathbf{b}}_{k+1}^{(-)}
\end{array}\right]=\left[\begin{array}{cc}
\mathbf{M}(\Delta \boldsymbol{\theta}) & \mathbf{0}_{4 \times 3} \\
\mathbf{0}_{3 \times 3} & \mathbf{I}_{3 \times 3}
\end{array}\right]\left[\begin{array}{l}
\hat{q}_{k}^{(+)} \\
\hat{\mathbf{b}}_{k}^{(+)}
\end{array}\right]
$$

In this case, the gyros' biases are considered constant on average, and its variation is considered as noise, hence it can 

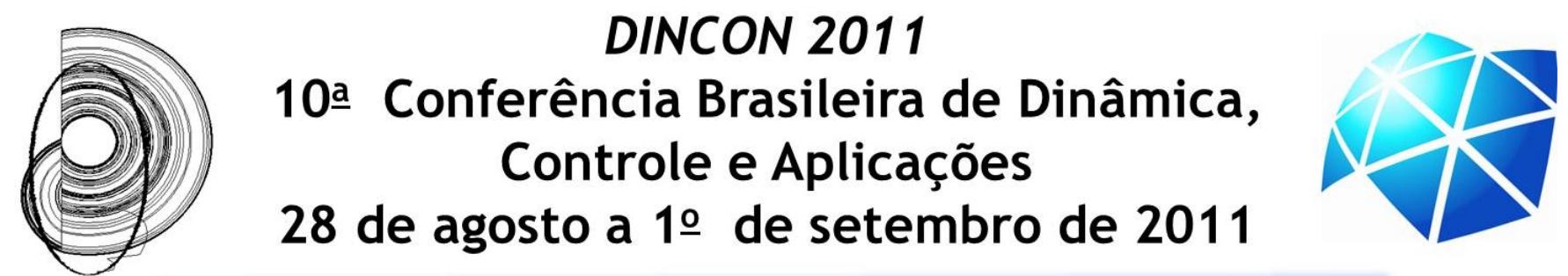

be propagated directly. In terms of the state vector and propagation matrix, the Eq. 7 can be rewritten as:

$$
\hat{\mathbf{x}}_{k+1}^{(-)}=\boldsymbol{\Phi}_{k} \hat{\mathbf{x}}_{k}^{(+)}
$$

Data quality after propagation depends essentially on the gyros' readout after removing their respective biases.

Before defining the propagation it is important to quote that the covariance matrix with dimension 7 is not numerically stable because its rank is 6 . To avoid numerical problems it is better to work with the reduced covariance, gain and measurement matrices. There is a reduction matrix to change from one form to another.

The Eq. 9 shows how the reduced covariance matrix is propagated. Note that the first term is a propagation of the covariance applying the reduced propagation matrix. This propagation does not propagate errors. Besides, the second term is a trapezoidal integral where the $\mathbf{Q}$ matrix is associated with the gyros noise and bias drift.

$$
\boldsymbol{\beta}_{k+1}^{6-)}=\boldsymbol{\Phi}_{k}^{0} \boldsymbol{\beta}_{k}^{\left.b^{+}\right)} \boldsymbol{\Phi}_{k}^{\top}+\left(\boldsymbol{\Phi}_{k}^{0} \boldsymbol{\phi}_{k}^{0} \boldsymbol{\phi}_{k}^{\top}+\boldsymbol{\phi}_{k}^{0}\right) \Delta t / 2
$$

The reduced transition matrix $\mathbf{\Phi}_{k}^{0}$ is calculated as shown in:

$$
\boldsymbol{\Phi}_{k}^{0}=\left[\begin{array}{cc}
\boldsymbol{\Lambda}_{k} & \mathbf{J}_{k} \\
\mathbf{0}_{3 \times 3} & \mathbf{I}_{3 \times 3}
\end{array}\right]
$$

The matrices $\boldsymbol{\Lambda}_{k}$ e $\mathbf{J}_{k}$ are:

$$
\begin{gathered}
\boldsymbol{\Lambda}_{k}=\mathbf{A}\left(\mathfrak{q}_{k+1}^{(-)}\right) \mathbf{A}^{\mathrm{T}}\left(\mathfrak{q}_{k}^{(+)}\right) \\
\mathbf{J}_{k}=-\left[\mathbf{A}\left(\mathfrak{q}_{k+1}^{(-)}\right) \mathbf{A}^{\mathrm{T}}\left(\mathfrak{q}_{k}^{(+)}\right)+\mathbf{I}_{3 \times 3}\right] \Delta t / 4
\end{gathered}
$$

Matrix $\mathbf{A}^{\mathrm{T}}$ is a rotation matrix equivalent to the quaternion, whose relationship is given by:

$$
\mathbf{A}(q)=\left(q_{0}^{2}-|\mathbf{q}|^{2}\right) \mathbf{I}_{3 \times 3}+2 \mathbf{q} \mathbf{q}^{\mathrm{T}}+2 q_{0} \mathbf{\Omega}_{3}(\mathbf{q})
$$

Term $q_{0}$ refers to the scalar component of the quaternion, and $\mathbf{q}$ refers to its vector component. Function $\Omega_{3}$ is the dimension 3 skew-symmetric operator.

\subsubsection{State and Covariance update}

State updating is done as shown:

$$
\hat{\mathbf{x}}_{k}^{(+)}=\hat{\mathbf{x}}_{k}^{(-)}+\mathbf{K}_{k}\left[\hat{q}_{\mathrm{ACS}}-\mathbf{H} \hat{q}_{k}^{(-)}\right]
$$

The greatest computational cost is that of computing the gain K. This gain could be computed through simulation and used as a constant. Updating of the reduced covariance matrix is done according to:

$$
\mathbf{P}_{k}^{\left.b^{+}\right)}=\mathbf{P}_{k}^{\left.b^{-}\right)}+\mathbf{K}_{k}^{0} \mathbf{H}_{k} \mathbf{P}_{k}^{(-)}
$$

The reduced Kalman gain can be calculated as:

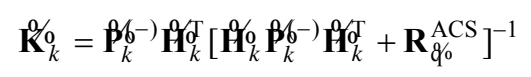

Where the matrix $\mathbf{H}_{k}$ is given by:

$$
\ddot{\mathbf{H}}_{k}=\mathbf{H}_{k} \mathbf{S}_{k}\left(\mathfrak{q}_{k}^{(-)}\right)
$$

The reduction matrix $\mathbf{S}_{k}\left(\mathcal{q}_{k}^{(-)}\right)$is a function of the current quaternion. Eq. 18 shows this function:

$$
\mathbf{S}_{k}(\mathcal{q})=\left[\begin{array}{ll}
\boldsymbol{\Xi}(\mathfrak{q}) & \mathbf{0}_{4 \times 3} \\
\mathbf{0}_{3 \times 3} & \mathbf{I}_{3 \times 3}
\end{array}\right]
$$

Where $\Xi(q)$ is the following distribution of the quaternion components in a matrix:

$$
\mathbf{\Xi}(\mathbf{q})=\left[\begin{array}{ccc}
q_{0} & -q_{3} & q_{2} \\
q_{3} & q_{0} & -q_{1} \\
-q_{2} & q_{1} & q_{0} \\
-q_{1} & -q_{2} & -q_{3}
\end{array}\right]
$$

The Kalman gain can be obtained from the reduced form gain by following relationship:

$$
\mathbf{K}_{k}=\mathbf{S}_{k}\left(\mathfrak{q}_{k}^{(-)}\right) \mathbf{K}_{k}
$$

\section{SIMULATION}

\subsection{Simulation Description}

MatLab simulations were performed to establish the filter convergence. Typical Gaussian noise was added to all sensors, and biases were added to the gyros. The gyros RMS noises were provided by the manufacturer [7].

The sampling rates for the gyros were set to $10 \mathrm{~Hz}$ and the attitude data readout rate was set to $1 \mathrm{~Hz}$, as expected in the actual implementation.

The attitude data and covariance was simulated generating data of two reference sensors (Sun and geomagnetic sensors) with their characteristics noises. The attitude was calculated using the TRIAD algorithm [8].

The simulation was performed considering two situations: (a) the body is static, with zero angular velocities; (b) the body has an angular velocity of $40 \mathrm{rpm}$ around $z$ axis. 


\subsection{Simulation Results}

In the first case the body is stopped. The Fig. 3 shows the convergence of errors in the gyros' biases and Fig. 4 shows the convergence of errors in the attitude quaternion's components.

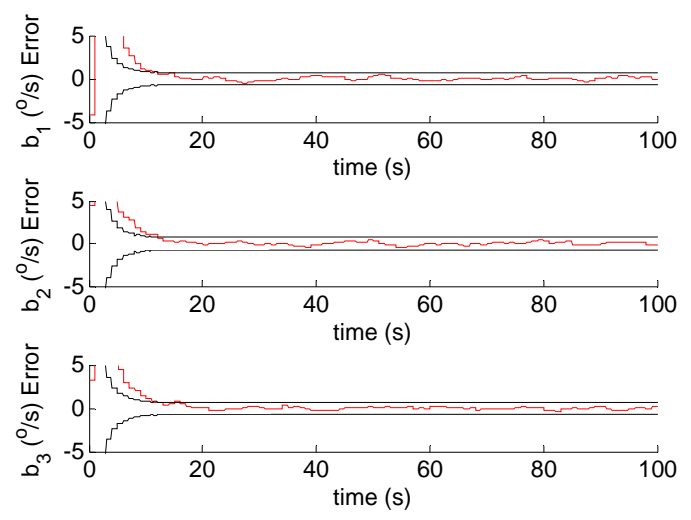

Fig. 3: Convergence of errors in the gyros' biases.
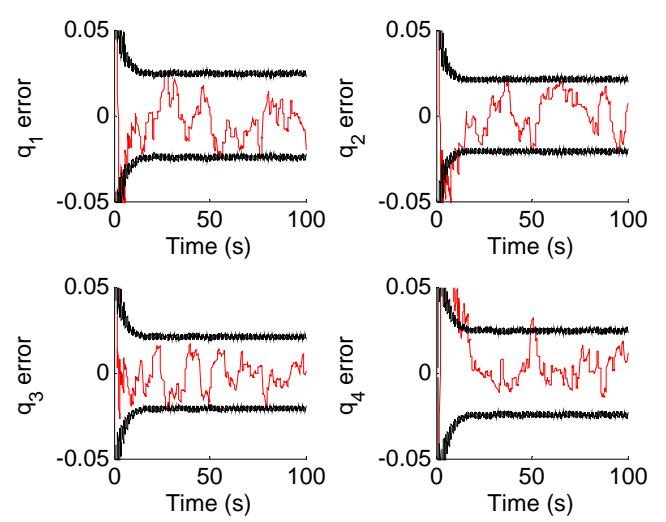

Fig. 4: Convergence of the attitude quaternion's components.

In the second case the body is turning around its $\mathrm{Z}$ axis. Fig. 5 shows the convergence of errors in the gyros' biases and Fig. 6 shows the convergence of errors in the attitude quaternion's components.

Statistically, there is a small advantage when redundant information such as the attitude propagated by the gyros and the attitude provided by the ACS are fused. The RMS noise is slightly smaller after filtering, but in this case the gyros' and the attitude sensors' noise were considered identical and equal to 0.01 RMS of each sensors' full scale.

\section{CONCLUSIONS}

This work performed an initial investigation about applying four tetrahedrally arranged gyrometers to propagate externally provided attitude information. The four angular velocities readings are converted into three orthogonal angular velocities on body axes and a Kalman Filter algorithm does the bias estimation on all axes and also estimates new attitude information considering the propagated attitude data. The Monte Carlo simulation results showed the filter convergence in a few seconds and also showed that bias variance is lower when attitude is constant, ie, the system is at rest. In general, the results are satisfactory and therefore the next step will be to implement the Kalman Filter algorithm on some embedded system using real sensor data.
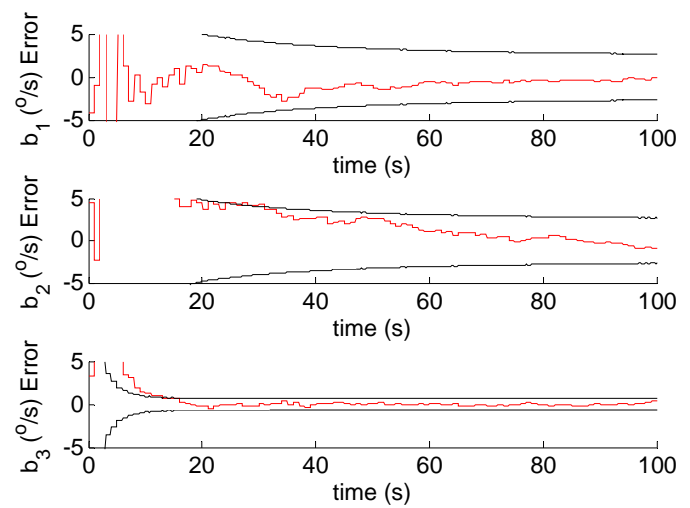

Fig. 5: Convergence of errors in the gyros' biases.
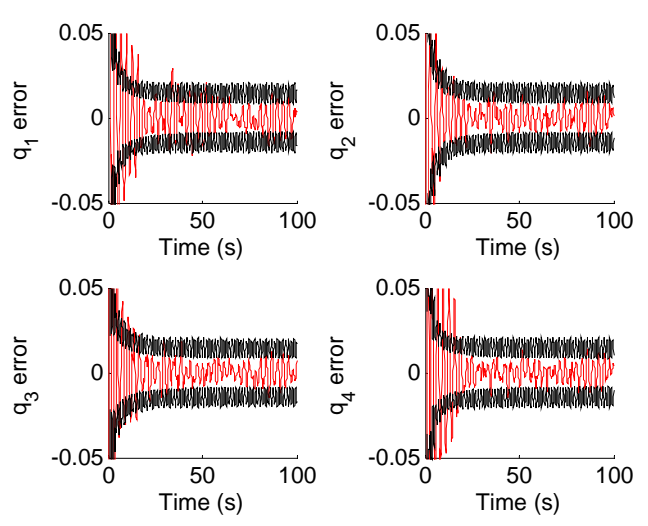

Fig. 6: Convergence of the attitude quaternion's components.

\section{REFERENCES}

[1]DOI Bogue, R.,"MEMS Sensors: past, present and future". Sensor Review v. 27, n. 1, 2007, pg. 7-13

[2] Tormena Jr, et al, "Development and Performance Analysis of an Autocalibration Method for Tri-axis Sensors in Attitude Estimation Systems", Proceedings of the XIV DINAME, 2011.

[3]PUB Vagner, M., "MEMS Gyroscope performance comparison using Allan Variance method". EEICT, 2011.

[4] Fernandes, David, 2009. "Satélite Tecnológico de Pequeno Porte ITASAT-1", I Workshop de Astronomia Espacial, <http:\|www.astro.iag.usp.br/ iwae>

[5]DOI Lefferts, E. J., Markley, F. L., Shuster, M. D.,1982. "Kalman filtering for spacecraft attitude estimation" J. Guidance, Reston, v.5, n.5, p.417-429.

[6] Granziera Jr., F., "Simulation and Implementation of a real-time atitude determination system using MEMS sensors", Master Thesis, DEEL/UEL, Londrina, 2006.

[7] Silicon Sense, "Datasheet of component SIRS01", $<$ http: $\mid w w w . s i l i c o n s e n s i n g . c o m / S i R R S 01>$

[8] Shuster, M. D., and S. D. Oh. Three-Axis Attitude Determination from Vector Observations. Journal of Guidance and Control 1980. Vol. 4, No. 1.pg. 70-77. 